\title{
Oral Galactose Provides a Different Approach to Incretin-Based Therapy of Alzheimer's Disease
}

\author{
Melita Salkovic-Petrisic*
}

Department of Pharmacology and Croatian Institute for Brain Research, University of Zagreb School of Medicine, Zagreb, Croatia

Article Info

\section{Article Notes}

Received: July 13, 2018

Accepted: August 21, 2018

\section{*Correspondence}

Dr. Salkovic-Petrisic Melita, Department of Pharmacology, University of Zagreb School of Medicine, Salata 11, HR-10 000 Zagreb, Croatia; Telephone No: +385 14590 219;

E-mail: melitas@mef.hr.

(C) 2018 Salkovic-Petrisic M. This article is distributed under the terms of the Creative Commons Attribution 4.0 International License

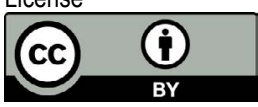

\section{Keywords}

Galactose

Sporadic Alzheimer's Disease

Streptozotocin

Glucagon-Like Peptide-1

Glucagon-Like Peptide-1 Receptor

Glucose-Dependent Insulinotropic Peptide

Incretin
Abstract

Incretin-based drugs originally developed for the treatment of type 2 diabetes mellitus are currently under investigation for their therapeutic potential in sporadic Alzheimer's disease (AD). Two major incretin hormones are glucagon-like peptide-1 (GLP-1) and glucose-dependent insulinotropic peptide (GIP), which both additionally have neuroprotective, neurotrophic and neurogenesis-promoting effects upon the stimulation of GLP-1 and GIP receptors in the brain. This review points out another approach to the incretin-related SAD therapy based on the therapeutic potential of oral galactose in a streptozotocin-induced rat model of sporadic AD (STZ-icv model). Chronic oral galactose treatment prevents the development and ameliorates already developed cognitive deficits in the early stage of SAD-like pathology in STZ-icv rat model. The underlying mechanism(s) of these beneficial effects might be related to stimulation of endogenous GLP-1-mediated central effects and normalization of cerebral glucose hypometabolism as well as other oral galactose-induced effects along the oro-gastro-intestinal tract. The beneficial effects on cognitive impairment seem to depend on galactose exposure, presence and stage of sAD-like pathology. Further research is needed to clarify therapeutic potential and safety profile of oral galactose treatment as well as its possible advantages over or synergistic activity with the GLP-1 analogues and inhibitors of dipeptidyl peptidase-4.

\section{Introduction}

Alzheimer's disease (AD) is the most common form of dementia for which there is no cure and no approved disease-modifying therapy, and which presents as a metabolic disorder of the brain characterised by insulin-resistant brain state (IRBS) ${ }^{1-3}$ and brain glucose hypometabolism ${ }^{4}$. Type 2 diabetes mellitus (T2DM) is a risk factor for $\mathrm{AD}^{5}$ which has increased the interest in restoring brain insulin signalling in $\mathrm{AD}$ with therapeutic agents originally developed for the treatment of T2DM, particularly those stimulating the action of glucagon-like peptide-1 (GLP-1) ${ }^{6,7}$.

GLP-1 and glucose-dependent insulinotropic peptide (GIP) are incretins, hormones released from the gut into the bloodstream in response to food and nutrient ingestion, which then stimulate $\beta$-cells in the pancreas to release insulin ${ }^{8,9}$. GLP-1 and GIP have important noninsulinotropic effects in extrapancreatic tissues, including the brain where both serve as neuromodulators demonstrating neuroprotective, neurotrophic and neurogenesis-promoting effects ${ }^{10,11}$. The actions of GLP-1 are mediated by GLP-1 receptors (GLP-1R) expressed in the gut, pancreas and other peripheral organs as well as throughout the brain $^{12}$. GLP-1 mimetics have shown promising results in animal models of neurodegenerative disorders, including $\mathrm{AD}^{13,14}$ and accomplished recently first positive results in $\mathrm{AD}$ clinical trials ${ }^{15}$.

Currently marketed GLP-1-based drugs are injectable GLP-1 
analogues, whose effects are mediated by activation of GLP$1 \mathrm{R}$, and oral inhibitors of dipeptidyl peptidase-4 (DPP4-I), the enzyme that rapidly inactivates circulating incretins ${ }^{16}$. Despite their multi-modality, based on their mechanism of action GLP-1R agonists and DPP4-I seem to be deprived of some beneficial effects that may contribute to the incretinbased therapy of neurodegenerative disorders. This review points out another approach to the incretin-related therapy based on the recent research on oral galactose therapeutic potential in a streptozotocin-induced rat model of $s A D^{17,18}$ (STZ-icv model) which develops cognitive deficits, IRBS and glucose hypometabolism in the brain ${ }^{19,20}$.

The rational for our research on oral galactose came from the previous work of Reutter and coworkers ${ }^{21}$ who found that galactose, a simple sugar composed of the same elements as glucose, is taken up by rat brain cells to a similar extent as by liver cells. In our preliminary work we wanted to explore whether drinking of small amounts of galactose solution ad libitum would prevent the cognitive deficits induced by STZ-icv treatment which reduces glucose metabolism in the brain ${ }^{17,20}$. Although modest, some clinical evidence supports the hypothesis that oral galactose might have beneficial effects on neuropathological processes, like in the case reports of congenital prosopagnosia, the lifelong inability to recognize people by their face ${ }^{22}$ and of a patient with inflammatory demyelinating brain lesions ${ }^{23}$. While in the former, remarkable improvement in face recognition, sense of orientation and mental activity vanished after discontinuation of oral galactose intake, in the latter, disappearance of numbness and amelioration of fatigue and central symptoms persisted 6 months after the therapy. Many clinical trials on galactose treatment indicate its harmless action and therapeutic effects in different pathological conditions although the cognition has not been explored in these trials.

The literature on the role of other simple sugars in the therapy of cognitive disorders is lacking and there are only a few reports indicating that they might have some beneficial effects on the brain. Some studies showed that fructose increased memory performance in rats ${ }^{24,25}$ and that it had positive effects on preservation of synaptic potential in guinea pig hippocampal slices ${ }^{26}$, in contrast to numerous studies demonstrating that fructose intake is linked with insulin resistance and cognitive impairment ${ }^{27}$. However, among sugars like fructose, lactose and saccharose which induced only partial neuroprotection, galactose $(+31.5$ $\mathrm{mmol} / \mathrm{l}$ ) most potently protected cholinergic neurons against hypoglycemia at low $\mathrm{pH}^{28}$.

D-galactose is a C-4 epimer of glucose, which is physiologically found only in very small quantities in the human and animal body. At high levels, induced by dysfunctional metabolism of galactose or by exogenous galactose load, galactose reacts with the free amines of amino acids in proteins and peptides and consequently forms advanced glycation end products which cause oxidative damage in the body ${ }^{29}$. These detrimental effects have been used for modelling of age-related development of brain oxidative stress and cognitive impairment following a chronic exposure of healthy mice or rats to parenteral galactose $^{30}$.

However, there are only a few studies of other groups on oral galactose effects in the brain at the molecular level. The research of Budni and coworkers ${ }^{31}$ showed that galactose given daily by oral gavage as a single bolus dose $(100 \mathrm{mg} / \mathrm{kg})$ increased the activity of mitochondrial respiratory chain complexes I, II, II-III and IV in the rat prefrontal cortex and hippocampus in the 1st, 2nd, 4th, 6th and 8th weeks of treatment. Since mitochondria is responsible for the generation of reactive oxygen species, the authors suggested that it cannot be ruled out that such a mitochondrial dysfunction may be, in part, a molecular mechanism involved in oral galactose effects, which could eventually contribute to the development of cognitive deficits observed after 6-8 weeks of treatment ${ }^{32}$. However, this seems to be contradictory to the studies with 6- to 8-week subcutaneous administration of galactose in mice which induced cognitive deficits but was associated with reduced levels of mitochondrial complex activity ${ }^{33-35}$. Whether the opposite effects might be species-dependent, since galactose-induced aging model was explored mostly in mice, or differ according to the stage of the pathology and its progression like suggested for the mitochondrial changes in $\mathrm{AD}$ patients ${ }^{36}$ remains to be determined. Despite that, it should be taken into account that all these preclinical studies on galactose effects, regardless the route of its administration were done in healthy animals with no underlying pathology.

One explanation why galactose might have beneficial effects in the pathological conditions like AD, which is associated with the hypoglicemic and acidic condition in the brain, could be related to the finding that galactose protected cholinergic neurons in hypoglycemia only at low $\mathrm{pH}$ levels between 6.7 and 6.9 but not at the physiological $\mathrm{pH}^{28}$. The reason for that might be in low $\mathrm{pH}$ necessary for optimal activity of enzymatic processes in metabolizing galactose as well as of the enzymes required in glycogen utilization since glycogen in the brain is the only endogenous fuel store capable of maintaining cerebral functions under hypometabolic conditions ${ }^{28}$. It could not be excluded that, since these processes are lacking or are reduced in the normal condition at the physiological $\mathrm{pH}$, more galactose is available to react and form advanced glycation end products and oxidative damage. Further research is needed to elucidate the difference in the mechanism of galactose action between physiological and pathological condition. 
Our research in STZ-icv rat model of SAD has shown that 1- to 2-month oral galactose treatment $(200 \mathrm{mg} / \mathrm{kg} /$ day, dissolved in water, drink ad libitum) prevented development of cognitive deficits when initiated immediately after the STZ-icv injection ${ }^{17}$, and improved already developed cognitive deficits when initiated 1 month after the STZ-icv injection ${ }^{18}$.

\section{Beneficial Effects of Oral Galactose}

Several factors along the path of ingested galactose should be considered as possible contributing factors to the beneficial central effects seen in the STZ-icv rat model of SAD.

\section{GLP-1- related effects}

Orally administered galactose enters the enterocytes by sodium glucose cotransporter SGLT ${ }^{37}$ (partly also by a transient insertion of glucose transporter 2 /GLUT2/ into the apical membrane ${ }^{38}$ ) and is transported out of the enterocytes by GLUT2 at the basolateral membrane ${ }^{37}$. The GLUT2 trafficking between apical and basolateral membrane is disturbed in insulin-resistant state $^{39}$. Galactose released from enterocytes is absorbed into the circulation (Figure 1b) and additionally, unlike the parenteral galactose, also acts in a paracrine fashion and stimulates adjacent endocrine L-cells to secrete GLP$1^{40}$ (Figure 1d). The majority of L-cells secreting GLP-1 are located in ileum and colon and have direct contact with orally given nutrients at their luminal surface, and with vascular tissue and parenterally given compounds through their basolateral surface ${ }^{41}$. Different structures/ signalling mechanisms and their regulations are present at the enterocyte luminal and basolateral membranes, respectively, which could account for different responses following oral and parenteral galactose (as well as any other drug) treatment ${ }^{38}$. Ileum-derived GLP- 1 acts as an incretin which dose-dependently promotes insulin secretion from the pancreatic beta cells (plasma peak values $15 \mathrm{~min}$ after nutrient ingestion) (Figure 1c), while colon-derived GLP1 might be the result of local neuronal signalling and is additionally proposed to serve as a messenger of the low energy levels leading to delayed increase of appetite or slowing of the gastrointestinal transit ${ }^{42}$.

Gut location-dependent functions of cells secreting GLP1 (L-cells) and GIP (K-cells, primarily upper intestine) might also contribute to the different response to oral galactose administered continuously as a drink ad libitum ${ }^{17,18}$ and as a single daily dose given via orogastric tube ${ }^{32,43}$. Unlike the bolus dose, smaller nutrient loads preferentially release both GLP-1 and GIP (Figure 1e) from the upper intestinal portion, associated also with smaller but continuous and more physiological daily rise in the incretin level. In line with that, our preliminary experiments showed that chronic oral galactose treatment also normalized STZicv-induced decrement in plasma GIP levels (manuscript in preparation). This allows a speculation that chronic oral galactose-induced effects in STZ-icv rat model might

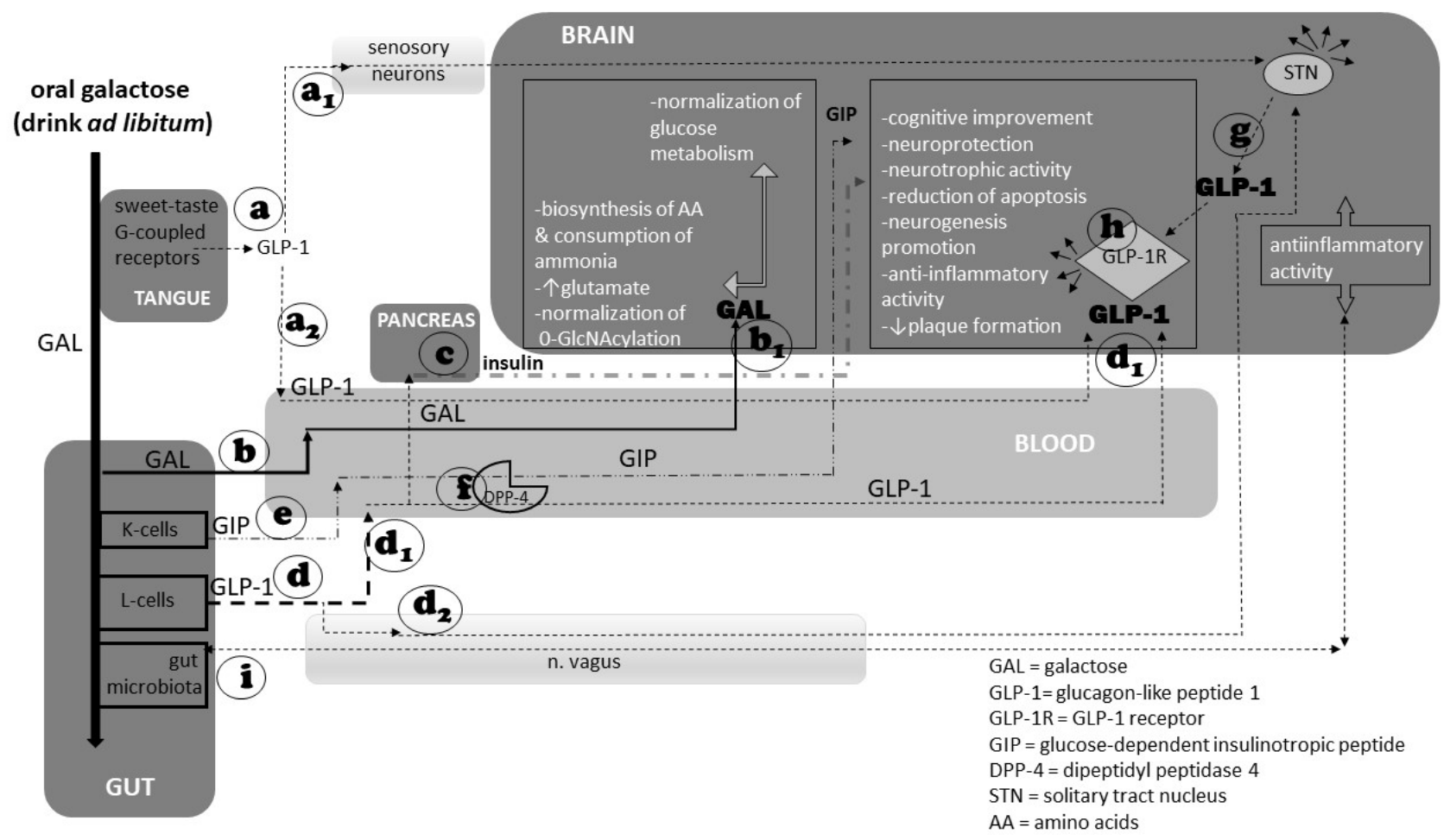

Figure 1: Proposed mechanisms of possible beneficial effects of oral galactose administered in small quantities as drink ad libitum. 
mimic the effects of dual GLP-1/GIP receptor agonist which demonstrates the neuroprotective effect in animal $\mathrm{AD}$ $\operatorname{model}^{44}$.

Additionally, continuous daily drink of galactose activates specific (G-protein coupled) sweet taste receptors in the tongue's taste buds (Figure 1a) and the gut, involved in regulation of GLP-1 secretion at both sites ${ }^{45}$. Small amounts of GLP-1 released in the tongue ${ }^{46}$, may send signals to the brain by activating adjacent sensory afferent neurons (Figure $1 a_{1}$ ) or by circulation (Figure $1 a_{2}$ ) and generating an additional stimulus that may contribute to the beneficial effects in the brain ${ }^{45}$. These effects are lacking when galactose is administered by orogastric tube as well as in the approved incretin-based therapy.

Our results demonstrated that the single oral galactose dose $(200 \mathrm{mg} / \mathrm{kg})$ tended to normalize the decrease in blood insulin levels seen transiently 1 month after STZ-icv treatment, but had no effects on normal insulin homeostasis observed in the chronic experiment in STZ-icv rat model ${ }^{18}$, suggesting that the beneficial activity of oral galactose on cognitive impairment in STZ-icv rats seems to be unrelated to the incretin effect.

Only $<25 \%$ of L-cell-secreted GLP-1 leaves the gut in its active form due to rapid inactivation by DPP4, expressed on the membranes of enterocytes, endothelial cells and throughout the brain ${ }^{9}$. Unlike the parenteral, oral administration of a single galactose dose $(200 \mathrm{mg} / \mathrm{kg})$ induced a huge $(+500 \%)$ increase in active GLP-1 blood levels (Figure $1 \mathrm{~d}_{1}$ ) in the STZ-icv but not in the control rats ${ }^{18}$. However, following a chronic oral galactose treatment, rise in active plasma GLP-1 levels normalized STZ-induced decrement to (but not over) the levels of galactoseuntreated controls ${ }^{18}$. Since active GLP-1 plasma levels were found unchanged in the galactose-untreated STZ-icv rats 1 month after icv injection but were significantly decreased after 3 months $^{18}$, it seems likely that GLP-1 degradation increases in the course of STZ-icv-induced pathology. The chronic oral galactose-induced increase observed only in the active but not in the total plasma GLP-1 levels in STZ-icv rat model, indirectly suggest that oral galactose might affect DPP4 inhibitory activity (Figure 1f), which could also contribute to the observed normalization of GIP plasma levels (manuscript in preparation). Considering the status of DPP4 in AD, literature data reveals no direct measurements of DPP4 activity in AD condition, but indicates that long-term inhibition of DPP4 in Alzheimerprone mice counteracts cognitive impairments ${ }^{47}$, and that DPP4-I have also therapeutic effects of on cognitive impairment in diabetic patients with or without $\mathrm{AD}^{48}$.

Intestinally released GLP-1 may also bind to and activate adjacent sensory afferents of the vagus nerve (Figure $1 d_{2}$ ), while in turn, the vagus may activate neurons of the solitary tract nucleus with a widespread projection pattern in the brainstem, hypothalamus, and forebrain, which consequently may also produce GLP-1 themselves ${ }^{49}$ (Figure $1 \mathrm{~g}$ ). The relative contribution of peripherally- versus centrally-derived GLP-1 in affecting the brain functions remains to be determined.

Peripherally- and centrally-derived GLP-1 activates GLP-1R in the brain located particularly on pyramidal neurons in the hippocampus and neocortex, on dendrites and cell bodies ${ }^{50}$. Activation of GLP-1R activates signalling pathways that converge with the insulin-signalling pathway and facilitate insulin signalling which may have beneficial effects on IRBS ${ }^{50}$. Oral galactose-induced cognitive improvement and increase in active plasma GLP-1 levels in STZ-icv rats are indeed followed by an increased expression of GLP-1R in the hippocampus ${ }^{18}$ (Figure $1 \mathrm{~h}$ ), which is in line with the enhancement of associative and spatial learning in mice following increments in GLP-1R expression in the hippocampus ${ }^{51}$. Another brain region rich in GLP-1R that may be significantly affected by GLP- 1 is the hypothalamus where a marked increase in GLP-1R expression was found following the oral galactose treatment in STZ-icv rats ${ }^{18}$, which might have further implications on hypothalamic regulation of neuroendocrine functions.

\section{Direct effects of circulating galactose on the brain}

Small quantities of absorbed galactose in the circulation escape liver degradation and reach the brain (Figure $1 b_{1}$ ), where they are transported into neurons by the insulin-independent GLUT3 in a concentrationdependent manner ${ }^{52}$. Our research has showed that oral galactose treatment increases the hippocampal GLUT3 expression $^{17,18}$. As already mentioned, the capacity of the brain to take up and metabolize galactose is similar to that of the liver ${ }^{21}$. Inside the cell galactose is quickly metabolized to glucose via the Leloir pathway ${ }^{53}$, suggesting that when intracellular glucose level/metabolism is decreased (like in SAD), galactose may serve as an alternative source of glucose/energy within the neurons. Our recent research on STZ-icv rat SAD model demonstrated that 2-month oral galactose treatment normalized STZ-induced glucose hypometabolism in the brain measured in vivo by the uptake of the positron emission tomography tracer $^{18}$ F-fluorodeoxyglucose ${ }^{18}$. However, additional involvement of GLP-1-related effects could not be excluded based on the GLP-1 analogue liraglutide-induced prevention of cerebral glucose metabolism decline in AD patients ${ }^{54}$.

There might be additional beneficial effects of circulating galactose in the brain. Similar to glucose, galactose in the brain is metabolized into amino acids leading to increased level of glutamate and gamma-aminobutyric acid ${ }^{21}$ which play important roles in normal cognitive functioning. Galactose has an advantage over glucose because the 
galactose metabolites persist for longer time in the brain ${ }^{21}$. The biosynthesis of amino acids from hexoses (via citric acid cycle) consumes equivalents of ammonia which is increased in $\mathrm{AD}^{55}$. Galactose-induced increment in glutamate levels may further be of importance considering the decreased glutamate levels found in the brain of $\mathrm{AD}$ patients ${ }^{56}$. However, such an increase should not go over the threshold of glutamatergic-induced excitotoxicity which is why low galactose exposure following oral, in contrast to parenteral administration, would be expected to have beneficial effects. Additionally, increased intracellular concentration of galactose or galactose-1-phosphate or UDP- galactose might also normalize 0-GlcNAcylation of the regulatory proteins, an important post-translation mode of regulation of protein functions, like in case of tau protein found compromised in $\mathrm{AD}^{57}$.

\section{Effects on gut microbiota}

Oral galactose may elicit effects in the gut before being absorbed by acting on intestinal microflora involved in bidirectional communication between the gut and the brain. Alterations in the amount/composition of gut microbiota might be linked to $\mathrm{AD}^{58}$ as evidenced by transgenic mice $\mathrm{AD}$ model, in which histological and cognitive $\mathrm{AD}$ manifestations were correlating with a specific gut microbiome state ${ }^{59}$. Microbiota dysbiosis increases the permeability of the gut and blood-brain barrier, and can induce inflammation but can also activate the vagus nerve and induce an anti-inflammatory reflex ${ }^{49,60}$ (Figure 1i). Consumption of a mixture of probiotics had a positive effect on cognitive function and some metabolic statuses in randomized, double-blind controlled clinical trials in AD patients ${ }^{61}$, while such a treatment attenuated age-related deficits in long-term potentiation in rats, suggesting an improvement in memory associated with amelioration of age-related microglial activation ${ }^{62}$. Unlike the glucose and fructose diets, nine weeks of oral galactose treatment (diet containing 15\% of dry matter) induced a favourable shift in rat gut microbial populations associated with improvement in hepatic insulin sensitivity ${ }^{63}$. Future research needs to elucidate the influence of oral galactose treatment on the gut microbiota in STZ-icv rat SAD model as a possible beneficial mechanism that is lacking in approved incretin-based drugs.

\section{Limitations of Oral Galactose Therapy}

It might be difficult to determine the exact level of galactose exposure associated with the beneficial effects in the $\mathrm{AD}$ brain since in healthy condition the high exposure induces detrimental effects which depend on age, gender and treatment duration ${ }^{30,31,43}$. The effects of oral galactose differ in the absence or presence of AD-like pathology (increase in both active plasma GLP-1 level and GLP-1R expression in the hippocampus of the STZ-icv rats versus decrease and no change of the respective parameters in healthy controls $)^{18}$. Moreover, they also seem to depend upon the animal model and stage of AD pathology with cognitive improvement seen only in the early and not advanced stage of SAD model, and not in transgenic (familial) AD mice model (manuscript submitted). The underlying molecular mechanisms involving GLP-1 and/or GIP and DPP4, and the contribution of each one separately, as well as the side-effects of the long-term oral galactose therapy or the persistency of the effects upon the therapy discontinuation, are still unclear.

\section{Conclusion}

Oral galactose treatment might offer a novel strategy to the AD therapy by combining several current trends; incretin-based therapy, nutrients as neuroprotective substances, and agent's multi-modality manifested here by stimulation of endogenous GLP-1-mediated central effects and by direct galactose effects in the brain and possibly along the oro-gastro-intestinal tract. Further research is needed to clarify therapeutic potential and safety profile of oral galactose as well as its possible advantages over or synergistic activity with the GLP-1 analogues/DPP4-I in the SAD treatment.

\section{Acknowledgement}

My associates in the Laboratory for Molecular Neuropharmacology are thanked for the dedicated work on oral galactose research. Supported by the Croatian Science Foundation (Project IP-2014-09-4639).

\section{References}

1. Hoyer S. Glucose metabolism and insulin receptor signal transduction in Alzheimer disease. Eur J Pharmacol. 2004. doi:10.1016/j. ejphar.2004.02.049

2. De Felice FG, Lourenco MV, Ferreira ST. How does brain insulin resistance develop in Alzheimer's disease? Alzheimers. Dement. 2014; 10: S26-32.

3. de la Monte SM, Tong M. Brain metabolic dysfunction at the core of Alzheimer's disease. Biochem. Pharmacol. 2014; 88: 548-59.

4. Mosconi L, Pupi A, De Leon MJ. Brain glucose hypometabolism and oxidative stress in preclinical Alzheimer's disease. Ann N Y Acad Sci. 2008; 1147: 180-195.

5. Leibson CL, Rocca WA, Hanson VA, et al. Risk of dementia among persons with diabetes mellitus: a population-based cohort study. Am J Epidemiol. 1997; 145: 301-8.

6. Holscher C. Incretin Analogues that have been Developed to Treat Type 2 Diabetes Hold Promise as a Novel Treatment Strategy for Alzheimers Disease. Recent Pat CNS Drug Discov. 2010; 5: 109-117.

7. Yarchoan M Arnold SE. Repurposing Diabetes Drugs for Brain Insulin Resistance in Alzheimer Disease. Diabetes. 2014; 63: 2253-2261.

8. Yabe D Seino Y. Two incretin hormones GLP-1 and GIP: Comparison of their actions in insulin secretion and $\beta$ cell preservation. Progress in Biophysics and Molecular Biology. 2011; 107: 248-256.

9. Holst JJ. The Physiology of Glucagon-like Peptide 1. Physiol Rev. 2007; 87: 1409-1439. 
10. Hölscher C. Central effects of GLP-1: New opportunities for treatments of neurodegenerative diseases. J Endocrinol. 2014; 221.

11. Figueiredo CP, Pamplona FA, Mazzuco TL, et al. Role of the glucosedependent insulinotropic polypeptide and its receptor in the central nervous system: therapeutic potential in neurological diseases. Behav Pharmacol. 2010; 21: 394-408.

12. Harkavyi A Whitton PS. Glucagon-like peptide 1 receptor stimulation as a means of neuroprotection. Br J Pharmacol. 2010; 159: 495-501.

13. Batista AF, Forny-Germano L, Clarke JR, et al. The diabetes drug liraglutide reverses cognitive impairment in mice and attenuates insulin receptor and synaptic pathology in a non-human primate model of Alzheimer's disease. J Pathol. 2018; 245: 85-100.

14. Cai HY, Yang JT, Wang ZJ, et al. Lixisenatide reduces amyloid plaques, neurofibrillary tangles and neuroinflammation in an APP/PS1/tau mouse model of Alzheimer's disease. Biochem. Biophys Res Commun. 2018; 495: 1034-1040.

15. Gejl M. In Alzheimer's Disease, Six-Month Treatment with GLP-1 Analogue Prevents Decline of Brain Glucose Metabolism: Randomized, Placebo-Controlled, Double-Blind Clinical Trial. Front Aging Neurosci. 2016; 8: 1-10.

16. Li Y, Li L, Hölscher C. Incretin-based therapy for type 2 diabetes mellitus is promising for treating neurodegenerative diseases. Rev Neurosci. 2016; 27: 689-711.

17. Salkovic-Petrisic M, Osmanovic-Barilar J, Knezovic A, et al. Longterm oral galactose treatment prevents cognitive deficits in male Wistar rats treated intracerebroventricularly with streptozotocin Neuropharmacology. 2014; 77: 68-80.

18. Knezovic A, Osmanovic Barilar J, Babic A, et al. Glucagon-like peptide-1 mediates effects of oral galactose in streptozotocin-induced rat model of sporadic Alzheimer's disease. Neuropharmacology. 2018; 135.

19. Salkovic-Petrisic M. Hoyer S. Central insulin resistance as a trigger for sporadic Alzheimer-like pathology: an experimental approach. J Neural Transm Suppl. 2007; 217-33.

20. Chen Y, Guo Z, Mao YF, et al. Intranasal Insulin Ameliorates Cerebra Hypometabolism, Neuronal Loss, and Astrogliosis in StreptozotocinInduced Alzheimer's Rat Model. Neurotox Res. 2018; 33: 716-724.

21. Roser M, Josic D, Kontou M, et al. Metabolism of galactose in the brain and liver of rats and its conversion into glutamate and other amino acids. J Neural Transm. 2009; 116: 131-139.

22. Esins J, Schultz J, Bülthoff I, et al. Galactose uncovers face recognition and mental images in congenital prosopagnosia: The first case report. Nutr Neurosci. 2014; 17: 239-240.

23. Panfoli I, Ravera S, Calzia D, et al. Missed evolution of demyelinizing brain lesions during supplementation with natural compounds: A Case Report. Med Res Arch. 2016; 3.

24. Messier C, White NM. Memory improvement by glucose, fructose, and two glucose analogs: a possible effect on peripheral glucose transport Behav Neural Biol. 1987; 48: 104-27.

25. Rodriguez WA, Horne CA, Mondragon AN, et al. Comparable doseresponse functions for the effects of glucose and fructose on memory. Behav Neural Biol. 1994; 61: 162-9.

26. Saitoh M, Okada Y, Nabetani M. Effect of mannose, fructose and lactate on the preservation of synaptic potentials in hippocampal slices. Neurosci Lett. 1994; 171: 125-8.

27. Moreira PI. High-sugar diets, type 2 diabetes and Alzheimer's disease. Curr Opin Clin Nutr Metab Care. 2013; 16: 440-445.

28. Pirchl M, Humpel C. Galactose Counteracts Hypoglycemia-Induced Decline of Cholinergic Neurons at Low pH in Organotypic Rat Brain Slices of the Basal Nucleus of Meynert. Pharmacology. 2011; 88: 245251.
29. Zhang XL, Jiang B, Li ZB, et al. Catalpol ameliorates cognition deficits and attenuates oxidative damage in the brain of senescent mice induced by d-galactose. Pharmacol Biochem Behav. 2007; 88: 64-72.

30. Cui X, Zuo P, Zhang Q et al. Chronic systemic D-galactose exposure induces memory loss, neurodegeneration, and oxidative damage in mice: Protective effects of R- $\alpha$-lipoic acid. J Neurosci Res. 2006; 83: 1584-1590.

31. Budni J, Garcez ML, Mina F, et al. The oral administration of D-galactose induces abnormalities within the mitochondrial respiratory chain in the brain of rats. Metab Brain Dis. 2017; 32: 811-817.

32. Budni J, Pacheco R, da Silva S, et al. Oral administration of D-galactose induces cognitive impairments and oxidative damage in rats. Behav Brain Res. 2016; 302: 35-43.

33. Kumar A, Dogra S, Prakash A. Effect of carvedilol on behavioral, mitochondrial dysfunction, and oxidative damage against d-galactose induced senescence in mice. Naunyn Schmiedebergs Arch Pharmacol. 2009; 380: 431-441.

34. Kumar A, Prakash A, Dogra S. Naringin alleviates cognitive impairment, mitochondrial dysfunction and oxidative stress induced by d-galactose in mice. Food Chem Toxicol. 2010; 48: 626-632.

35. Shwe T, Pratchayasakul W, Chattipakorn N, et al. Role of D-galactoseinduced brain aging and its potential used for therapeutic interventions. Exp Gerontol. 2018; 101: 13-36.

36. Gibson GE, Shi Q. A Mitocentric View of Alzheimer's Disease Suggests Multi-Faceted Treatments. J Alzheimer's Dis. 2010; 20: S591-S607.

37. Wright EM, Martín MG, Turk E. Intestinal absorption in health and disease - Sugars. Bailliere's Best Practice and Research in Clinical Gastroenterology. 2003; 17: 943-956.

38. Kellett GL, Brot-Laroche E, Mace OJ, et al. Sugar absorption in the intestine: the role of GLUT2. Annu Rev Nutr. 2008; 28: 35-54.

39. Tobin V, Le Gall M, Fioramonti X, et al. Insulin Internalizes GLUT2 in the Enterocytes of Healthy but Not Insulin-Resistant Mice. Diabetes. $2008 ; 57$.

40. Ritzel U, Fromme A, Ottleben M, et al. Release of glucagon-like peptide-1 (GLP-1) by carbohydrates in the perfused rat ileum. Acta Diabetol. 1997; 34: 18-21.

41. Candeias EM, Sebastião IC, Cardoso SM, et al. Gut-brain connection: The neuroprotective effects of the anti-diabetic drug liraglutide. World J Diabetes. 2015; 6: 807-27.

42. Greiner TU, Bäckhed F. Microbial regulation of GLP-1 and L-cell biology. Molecular Metabolism. 2016; 5: 753-758.

43. Chogtu B, Arivazhahan A, Kunder SK, et al. Evaluation of acute and chronic effects of D-galactose on memory and learning in Wistar rats. Clin Psychopharmacol Neurosci. 2017.

44. Shi L, Zhang Z, Li L, et al. A novel dual GLP-1/GIP receptor agonist alleviates cognitive decline by re-sensitizing insulin signaling in the Alzheimer icv. STZ rat model Behav Brain Res. 2017; 327: 65-74.

45. Lee A, Owyang C. Sugars, Sweet Taste Receptors, and Brain Responses. Nutrients. 2017; 9: 653.

46. Feng XH, Liu XM, Zhou LH, et al. Expression of glucagon-like peptide-1 in the taste buds of rat circumvallate papillae. Acta Histochem. 2008; 110: 151-154.

47. D’Amico M, Di Filippo C, Marfella R, et al. Long-term inhibition of dipeptidyl peptidase-4 in Alzheimer's prone mice. Exp Gerontol. 2010; 45: 202-207.

48. Isik AT, Soysal P, Yay A, et al. The effects of sitagliptin, a DPP-4 inhibitor, on cognitive functions in elderly diabetic patients with or without Alzheimer's disease. Diabetes Res Clin Pract. 2017; 123: 192-198.

49. Forsythe P, Bienenstock J, Kunze WA. Vagal pathways for microbiome- 
brain-gut axis communication. Advances in experimental medicine and biology. 2014; 817: 115-133.

50. Gu G, Roland B, Tomaselli K, et al. Glucagon-like peptide-1 in the rat brain: Distribution of expression and functional implication. J Comp Neurol. 2013; 521: 2235-2261.

51. During MJ, Cao L, Zuzga DS, et al. Glucagon-like peptide-1 receptor is involved in learning and neuroprotection. Nat Med. 2003; :, 1173-1179.

52. Seatter MJ, Kane S, Porter LM, et al. Structure-Function Studies of the Brain-Type Glucose Transporter, GLUT3: Alanine-Scanning Mutagenesis of Putative Transmembrane Helix VIII and an Investigation of the Role of Proline Residues in Transport Catalysis †. Biochemistry. 1997; 36: 6401-6407.

53. Cohn RM, Segal S. Galactose metabolism and its regulation. Metabolism. 1973; 22: 627-642.

54. Gejl M, Gjedde A, Egefjord L, et al. No decline of brain glucose metabolism in Alzheimer's disease patients treated with liraglutide. Diabetes. 2015; 64: A340.

55. Seiler N. Ammonia and Alzheimer's disease. Neurochem Int. 2002; 41 189-207.

56. Fayed N, Modrego PJ, Rojas-Salinas G, et al. Brain Glutamate Levels Are Decreased in Alzheimer's Disease. Am J Alzheimer's Dis Other Dementiasr. 2011; 26: 450-456.
57. Yang X, Qian K. Protein 0-GlcNAcylation: emerging mechanisms and functions. Nat Rev Mol Cell Biol. 2017; 18: 452-465.

58. Vogt NM, Kerby RL, Dill-McFarland KA, et al. Gut microbiome alterations in Alzheimer's disease. Sci Rep. 2017; 7: 13537.

59. Shen L, Liu L, Ji HF. Alzheimer's disease histological and behavioral manifestations in transgenic mice correlate with specific gut microbiome state. J Alzheimer's Dis. 2017; 56: 385-390.

60. Fung TC, Olson CA, Hsiao EY. Interactions between the microbiota, immune and nervous systems in health and disease. Nature Neuroscience. 2017; 20: 145-155.

61. Akbari E, Asemi Z, Daneshvar Kakhaki R, et al. Effect of Probiotic Supplementation on Cognitive Function and Metabolic Status in Alzheimer's Disease: A Randomized, Double-Blind and Controlled Trial. Front Aging Neurosci. 2016; 8: 256.

62. Distrutti E, O'Reilly JA, McDonald C, et al. Modulation of intestinal microbiota by the probiotic VSL\#3 resets brain gene expression and ameliorates the age-related deficit in LTP. PLoS One. 2014; 9.

63. Stahel P, Kim JJ, Xiao C. Of the milk sugars, galactose, but not prebiotic galacto-oligosaccharide, improves insulin sensitivity in male SpragueDawley rats. PLoS One. 2017; 12 